\title{
Design of Highway Alignment
}

\author{
${ }^{1}$ P. Nimitha, ${ }^{2}$ Malik Fahad, ${ }^{3}$ Mohammmad Abdul Dastagir Ahmed \\ ${ }^{4} \mathrm{C}$. Sindhu, ${ }^{5} \mathrm{G}$. Mounika. \\ ${ }^{1}$ Assistant professor, Civil Engineering Department, SVS Group Of Institutions, JNTUH Affiliated, Telangana, \\ INDIA. \\ ${ }^{2}$ Civil Engineering Department, SVS Institute of technology and science, JNTUH Affiliated, Telangana, INDIA \\ ${ }^{3}$ Civil Engineering Department, SVS Institute of technology and science, JNTUH Affiliated, Telangana, INDIA \\ ${ }^{4}$ Civil Engineering Department, SVS Institute of technology and science, JNTUH Affiliated, Telangana, INDIA \\ ${ }^{4}$ Civil Engineering Department, SVS Institute of technology and science, JNTUH Affiliated, Telangana, INDIA
}

\begin{abstract}
To establish the alignment of a highway of length $1 \mathrm{~km}$ from "huzurabad to parkal" for extension of highway from two way lane to four way lane and evaluation of soil strength on the field in the form of an open transverse. The main objective of the project is to design a Highway Alignment from "Huzurabad to Parkal" for extension of highway from two way lane to four way lanes and evaluation of soil strength on field. It is an existing road already but now its road width is extended so our aim is to design the highway alignment by the following steps:

1. To carry out the detailed profile leveling which includes longitudinal sectioning and cross sectioning.

2. To fix up the formation level from the plotted longitudinal section according to prevailing gradients.

3. The earthwork has been calculated and the same is present in the graphical format.

4. Collecting the soil samples from the site and testing it in the laboratory manually.

We used theodolites, chain, arrows, tape, ranging rods, tripod, levelling staff.

Highway Alignment is the position or layout of centre line of the highway on the ground is called the alignment. it includes straight path, horizontal deviation and curves.
\end{abstract}

Keywords: Highway alignment, longitudinal sectioning, cross sectioning, earthwork calculations.

\subsection{Highway Alignment:}

\section{Introduction}

The position or the layout of the centre line of the highway on the ground is called the alignment. Alignments surveys are widely provided for safety and protection purposes of different routes and highways. The alignment surveys include horizontal alignment, cross section angle and vertical alignment of roads. The process of alignment is available as most important and crucial process for road safety. Different kinds of advanced and innovative techniques are used to provide the alignment survey. The alignment survey of road is not carried out in an easy and quick manner.

A new road should be aligned very carefully as improper alignment would result in one or more of the following disadvantages:

a) Increase in construction cost

b) Increase in maintenance cost

c) Increase in vehicle operation cost

d) Increase in accident rate

Once the road is aligned and constructed, it is not easy to change the alignment due to increase in cost of adjoining land and construction of costly structures by the road side.

\subsection{Types of Alignment:}

These are two types of alignments. They are as follow:

1) Horizontal alignment

2) Vertical alignment

\subsection{Horizontal Alignment:}

The operational characteristics of a roadway are directly affected by the horizontal alignment. The designer must consider the roads terrain, traffic volume, expected capacity and LOS together with other safety factors in order to properly anticipate the posted speed. Highways will be designed according to their anticipated posted speed as opposed to an arbitrary design speed. 


\subsection{Vertical Alignment:}

Roadway vertical alignment is controlled by design speed, topography, traffic volumes and composition, highway functional classification, safety, sight distance, typical sections, horizontal alignment, climate, vertical clearances, drainage, economics, and aesthetics. When a highway is located where environmental resources exist the vertical alignment should be designed to minimize impacts. Vertical alignment should be properly coordinated with the natural topography, available right-of-way, utilities, roadside development and natural and man-made drainage patterns.

\subsection{Objectives of Present Study:}

In the present study a road of length $1 \mathrm{~km}$ located at "KANDUGULA VILLAGE, HUZURABAD MANDAL, KARIMNAGAR DISTRICT".

The objectives of present study are:

a) To carry out detailed profile levelling which includes longitudinal sectioning and cross sectioning. Longitudinal sectioning has been carried out at $10 \mathrm{~m}$ interval and cross sectioning has been carried out for a width of $7 \mathrm{~m}$.

b) To fix up the formation level from the plotted longitudinal section according to prevailing gradients, then the earth work has been calculated and the same is present in the graphical format called "Mass Diagram" or "Mass Curve".

c) To design the horizontal alignment which mainly comprises of horizontal circular curve and its related geometric elements like camber, super elevation along with transition curve and an attempt has also been made to design the vertical alignment which mainly comprises of vertical curves. Depending upon the gradient obtained in longitudinal sectioning a valley curve or summit curve has been designed.

d) To collect soil samples at zero meter chainage and $750 \mathrm{~m}$. Chainage as the change in soil condition has been observed

\subsection{Highway Project:}

\section{Literature Review}

General: In the new highway project, the engineer has to plan, design and commie: either a net-work of new roads or a road link. There are also projects requiring re-design and re-alignment of existing roads for upgrading the geometric design standards.

Once a highway is constructed, development takes place along the adjoining land and subsequent changes in alignment or improvements in geometric standards become very difficult. A badly aligned highway is not only a source of potential traffic hazard, but also causes a consideration increases in transportation cost and strain on the drivers and passengers. Therefore, proper investigation and planning are most important in a road project, keeping in view the present day needs as well as the future developments of the region.

\subsection{New Highway Project:}

The new highway project work may be divided into following stages:

- Selection of route, finalization of highway alignment and geometric design details.

- Collection of materials and testing of sub grade soil and design details of pavement layers.

- Construction stages including quality control.

\section{Route selection:}

The selection of route is made keeping in view the requirements of alignment and the geological, topographical and other features of the locality. However special care should be taken as regards the geometric design standards of the road for possible upgrading of speed standards in future, without being necessary to realign the road. After the alignment is finalized, the plans and working drawings are prepared.

\subsection{Plotting of longitudinal section:}

\section{Profile Levelling}

Profile levelling is the process of determining the elevations of points at short measured intervals along a fixed line such as the centre line of a railway, highway, canal or sewer. The fixed line may be a single straight line or it may be composed of a succession of straight lines or of a series of straight lines connected by curves. It is also known as longitudinal sectioning. By means of such sections the engineer is enabled to study the relationship between the existing ground surface and the levels of the proposed construction in the direction of its length. The profile is usually plotted on specially prepared profile paper on which the vertical scale is much larger than the horizontal and on this profile various studies relating to the fixing of grades and the estimating of costs are made. 


\subsection{Plotting of cross section:}

Cross sections are run at right angles to the longitudinal profile and on either side of it for the purpose of lateral outline of the ground surface. They provide the data for estimating quantities of earth work and for other purposes. The cross sections are numbered consecutively from the commencement of the centre line and are set out at right angles to the main line of section with the chain and tape, the cross staff or the optical square and the distances are measured left and right from the centre peg cross section may be taken at each chain. The length of cross section depends upon the nature of work.

\subsection{Earthwork:}

General:

Cross section of earth work of road in banking or in cutting is usually in the form of trapezium, and quantity of earthwork may be calculated by the following methods:

Quantity or volume $=$ sectional area $\mathrm{x}$ length

Sectional area $=$ area of central rectangular portion + area of two side triangular portions

$$
=\mathrm{BD}+2\left(\frac{1}{2 s d} \mathrm{~d}\right)=\mathrm{Bd}+\mathrm{sd}^{2}
$$

S:I is the ratio of side slopes as horizontal: vertical. For 1 vertical, horizontal is $\mathrm{s}$, for $\mathrm{d}$ vertical, horizontal is sd.

$$
\text { Quantity }=\left(B d+\mathrm{Sd}^{2}\right) \times \mathrm{L}
$$

When the ground is in a longitudinal slope. The height of bank or the depth of cutting will be different at the two ends of the section, and mean height or depth may be taken for ' $\mathrm{d}$ ' and sectional area at mid section is taken out for mean height. Alternatively, sectional area at the two ends may be calculated and the mean of two sectional areas is taken out.

Mean height $=\left(d_{1}+d_{2}\right) / 2$

In the present study mid sectional area method is used which is explained below.

\section{Mid sectional area method:}

Quantity $=$ area of mid section $\mathrm{x}$ length.

Let $\mathrm{d} 1 \& \mathrm{~d} 2$ be the height of bank at two ends portion of embankment, $\mathrm{L}$ the length of the section, $\mathrm{B}$ the formation width and 8:1 the side slope then,

Area of mid section $=$ Area of rectangular portion + area of two triangular portion

$$
=\mathrm{Bdm}=\frac{1}{2} \mathrm{sdm}^{2}+\frac{1}{2 \mathrm{sdm}^{2}}=\mathrm{Bdm}+\mathrm{sdm}^{2}
$$

Quantity of earthwork $=\left(B d m+s d m^{2}\right) \times L$

General $\mathrm{Q}=\left(\mathrm{Bd}+\mathrm{sd}^{2}\right) \mathrm{x} \mathrm{L}$, where $\mathrm{d}$ stands for mean height or depth.

\section{Mass Diagram or Mass Curve:}

The earth work for a highway involves cuts and fills. The most balanced design is one which utilities the cut volume in adjacent fills. This is achieved by a mass diagram which is a graphical representation of the amount of cut and the amount of fill and the manner in which the earth is to be hauled from cut to fill.

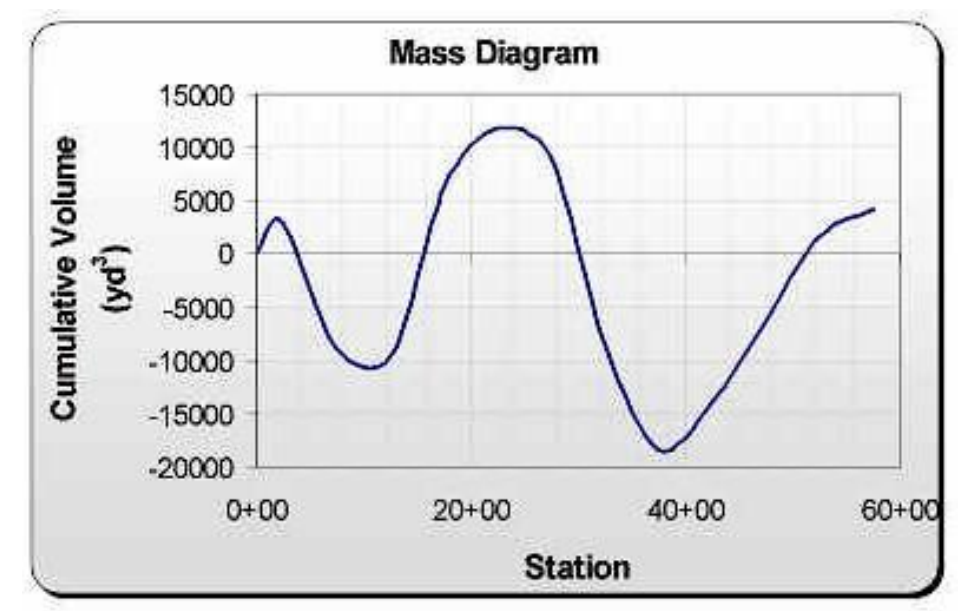

Fig 1- Mass Diagram 


\subsection{Design of Horizontal Alignment: \\ Horizontal Curve:}

The radius of horizontal curves is calculated by the relation

$$
\mathrm{R}=\mathrm{V}^{2} / 127(\mathrm{e}+\mathrm{f})
$$

$\mathrm{V}=$ Design speed

$\mathrm{e}=$ Design super-elevation rate

$\mathrm{f}=$ Transverse coefficient of friction $=0.15$

A horizontal highway curve is a curve in plan to provide change in direction to be central line of road. When a vehicle traverses a horizontal curve, the centrifugal force acts horizontally outwards through the centre of gravity of vehicles. The centrifugal force developed depends on the radius of the horizontal curves and the speed o the vehicles negotiating the curve. This centrifugal force is countered by the transverse friction resistance developed between the tyres and the pavement which enables the vehicles changes the direction along the curve and maintains the stability of the vehicles.

Centrifugal force $\mathrm{p}$ is given by the equation:

$$
\mathrm{p}=\frac{W V^{2}}{g R}
$$

Here,

$\mathrm{p}=$ Centrifugal force, $\mathrm{k}$

$\mathrm{W}=$ Weight of the vehicle, $\mathrm{kg}$

$\mathrm{R}=$ Radius of the circular curve, $\mathrm{m}$

$\mathrm{V}=$ Speed of the vehicles, $\mathrm{m} / \mathrm{sec}$

The ratio of the centrifugal force to the weight of the vehicle, $\mathrm{P} / \mathrm{W}$ is known as the centrifugal ratio or the impact factor. The centrifugal ration is thus equal to $\frac{V^{2}}{g R}$

The centrifugal force acting on a vehicle negotiating a horizontal curve has two effects:

i. Tendency to overturn the vehicle outwards about the outer wheels and

ii. Tendency to skid vehicles laterally outwards.

\section{a) Overturning effects:}

The centrifugal force that tends the vehicle to overturn about the outer wheels on a horizontal curve without super elevation is illustrated. The overturning moment due to centrifugal force $\mathrm{P}$ is $\mathrm{Px}$; this is resisted by the restoring moment due to weight of the vehicle $\mathrm{W}$ and is equal to $\mathrm{w}, \mathrm{b} / 2$, where $\mathrm{h}$ is the height of the centre of gravity of the vehicle above the road surface and $b$ is the width of the wheel base or the wheel track of the vehicle.

\section{b) skidding effect:}

The centrifugal forces developed as also the tendency to push the vehicles outwards in the transverse direction. If the centrifugal force $p$ developed excess the maximum possible transverse skid resistance due to the friction, the vehicles will start skidding in the transverse direction. The equilibrium condition for the transverse skid resistance developed is given by:

$$
\mathrm{P}=\mathrm{Fa}+\mathrm{Fb}=\mathrm{f}(\mathrm{Ra}+\mathrm{Rb})=\mathrm{Fw}
$$

In the above relation $\mathrm{f}$ is the coefficient of friction between the tyre and the pavement surface in the transverse direction, $\mathrm{Ra}$ and $\mathrm{Rb}$ are normal reactions at the wheels $\mathrm{A}$ and $\mathrm{B}$ such that $(\mathrm{Ra}+\mathrm{Rb})$ is equal to the weight $\mathrm{W}$ of the vehicles, as no super elevation has been provided in this case.

Since $\mathrm{P}=\mathrm{fW}$, the centrifugal ratio $\mathrm{P} / \mathrm{W}$ is equal to ' $\mathrm{f}$ '. Thus to avoid overturning and lateral skidding on a horizontal curve, the centrifugal ratio should always be less than $b / 2 h$ and also ' $\mathrm{'}$ '.

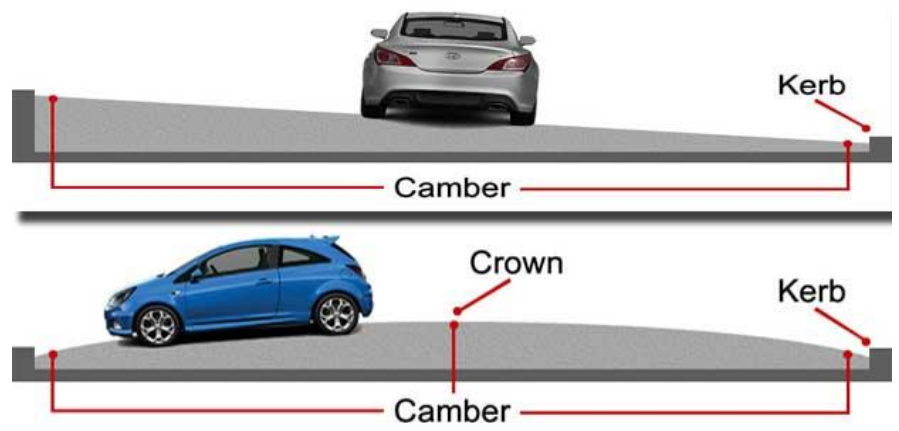

Fig 2- Camber 


\section{Data Collection And Survey Methodology}

The objectives of the present study needs the following type of survey.

- $\quad$ Profile levelling

\subsection{Profile Levelling:}

This type of survey mainly consist of

a. Longitudinal sectioning \&

b. Cross sectioning

\section{Longitudinal Sectioning and Cross-Sectioning:}

Longitudinal sectioning is used in route surveys for highways and railways. The reduced levels of points along the proposed centre line, a known (usually regular) distance apart are obtained by the principles of differential and fly levelling. The objects are to determine the profile of the ground in a vertical longitudinal sectioning. It is the process of determining the reduced levels of points at measured intervals along a chosen alignment of a line. During the location of highways, railways, canals and sewers, stakes or pegs are placed ill regular intervals along the proposed centre line, and the levels of the ground at these points It determined from convenient level stations on either side of it. The peg intervals is commonly $30 \mathrm{~m}$, but may be reduced to $10 \mathrm{~m}$ to $15 \mathrm{~m}$ in rough terrain. In addition, levels are taken at points where a change in direction occurs, at breaks in the slope of the ground surface, and at critical points like culverts, bridges and other features crossing the alignment.

When plotted, these levels show a profile, a line depicting elevations of ground points one longitudinal vertical section along the alignment. Since distances must be measured, the moment includes chain or tape levelling instrument such as Auto level, along with a levelling staff. Apart from the columns for noting staff readings and reduced levels, additional whims in the level field book are required to record the designation of the points, Longitudinal sectioning has been carried out at $10 \mathrm{~m}$ intervals throughout the length of alignment. Cross sectioning has been carried out for $7 \mathrm{~m}$ width i.e., $3.5 \mathrm{~m}$ on left of centre line and $3.5 \mathrm{~m}$ on right of centerline.

\section{Longitudinal Sectioning:}

Table 1: Longitudinal section of road

\begin{tabular}{|c|c|c|c|c|c|}
\hline CHAINAGE & B.S & I.S & F.S & H.I & R.L \\
\hline & 1.30 & & & 101.30 & 100.00 \\
\hline 0.00 & & 1.81 & & 101.30 & 99.49 \\
\hline 10.00 & & 2.20 & & 101.30 & 99.10 \\
\hline 20.00 & & 2.39 & & 101.30 & 98.91 \\
\hline 30.00 & & 2.31 & & 101.30 & 98.99 \\
\hline 40.00 & & 2.09 & & 101.30 & 99.21 \\
\hline 50.00 & & 2.08 & & 101.30 & 99.22 \\
\hline 60.00 & & 2.01 & & 101.30 & 99.29 \\
\hline 70.00 & & 2.19 & & 101.30 & 99.11 \\
\hline 80.00 & & 2.20 & & 101.30 & 99.10 \\
\hline 90.00 & & 1.42 & & 101.30 & 99.88 \\
\hline 100.00 & & 1.50 & & 101.30 & 99.80 \\
\hline 110.00 & & 1.57 & & 101.30 & 99.73 \\
\hline 120.00 & & 1.59 & & 101.30 & 99.71 \\
\hline 130.00 & & 1.62 & & 101.30 & 99.68 \\
\hline 140.00 & & 1.64 & & 101.30 & 99.66 \\
\hline 150.00 & & 1.65 & & 101.30 & 99.65 \\
\hline 160.00 & & 1.66 & & 101.30 & 99.64 \\
\hline 170.00 & & 1.68 & & 101.30 & 99.62 \\
\hline 180.00 & & 1.56 & & 101.30 & 99.74 \\
\hline 190.00 & & 1.52 & & 101.30 & 99.78 \\
\hline 200.00 & & 1.50 & & 101.30 & 99.80 \\
\hline 210.00 & 1.50 & & & 101.50 & 100.00 \\
\hline 220.00 & & 1.54 & & 101.50 & 99.96 \\
\hline 230.00 & & 1.55 & & 101.50 & 99.95 \\
\hline 240.00 & & 1.57 & & 101.50 & 99.93 \\
\hline 250.00 & & 1.59 & & 101.50 & 99.91 \\
\hline 260.00 & & 1.60 & & 101.50 & 99.90 \\
\hline 270.00 & & 1.63 & & 101.50 & 99.87 \\
\hline 280.00 & & 1.64 & & 101.50 & 99.86 \\
\hline 290.00 & & 1.65 & & 101.50 & 99.85 \\
\hline 300.00 & & 1.74 & & 101.50 & 99.76 \\
\hline 310.00 & & 1.82 & & 101.50 & 99.68 \\
\hline 320.00 & & 2.21 & & 101.50 & 99.29 \\
\hline 330.00 & & 2.32 & & 101.50 & 99.18 \\
\hline
\end{tabular}




\begin{tabular}{|c|l|l|l|l|l|}
\hline 340.00 & & 2.33 & & 101.50 & 99.17 \\
\hline 350.00 & & 2.20 & & 101.50 & 99.30 \\
\hline 360.00 & & 2.25 & & 101.50 & 99.25 \\
\hline 370.00 & & 2.32 & & 101.50 & 99.18 \\
\hline 380.00 & & 2.35 & & 101.50 & 99.15 \\
\hline 390.00 & & 2.36 & & 101.50 & 99.14 \\
\hline 400.00 & & 2.38 & & 101.50 & 99.12 \\
\hline 410.00 & & 2.41 & & 101.50 & 99.09 \\
\hline 420.00 & & 2.32 & & 101.50 & 99.18 \\
\hline 430.00 & & 2.22 & & 101.50 & 99.28 \\
\hline 440.00 & & 2.27 & & 101.50 & 99.23 \\
\hline 450.00 & & 2.31 & & 101.50 & 99.19 \\
\hline 460.00 & & 2.35 & & 101.50 & 99.15 \\
\hline 470.00 & & 1.80 & & 101.50 & 99.70 \\
\hline 480.00 & & 1.70 & & 101.50 & 99.80 \\
\hline 490.00 & & 1.50 & & 101.50 & 100.00 \\
\hline 500.00 & 1.50 & & & 101.50 & 100.00 \\
\hline & & & & &
\end{tabular}

\section{Cross-Sectloning:}

The cross section of a road is a vertical plane at right angles to the road control line. It is viewed in the direction of increasing stationing and shows transverse detail of the various elements that make up the road's structure, sometimes from boundary to boundary. The main purpose of a cross section is to show the variation of elements within the design and their interaction with the natural topography. The cross section elements should be designed to ensure that the use of the space available within the road reserve is sympathetic to the natural environment and user expectations, while maintaining a balance between construction, maintenance and operating (including crash) costs.

CROSS SECTION OF A ROAD

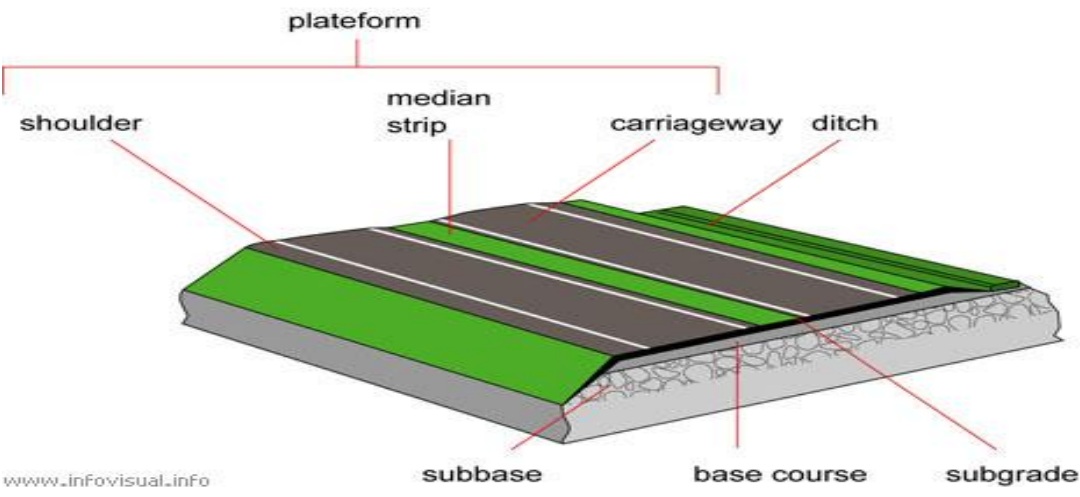

Table 2- Cross Section of Road

\begin{tabular}{|c|c|c|c|c|c|}
\hline $\begin{array}{c}\text { CHAI- } \\
\text { NAGE }\end{array}$ & LEFT & RIGHT & $\begin{array}{c}\text { STAFF } \\
\text { READING }\end{array}$ & H.I & R.L \\
\hline 0.00 & 3.5 & & 1.46 & 101.3 & 99.84 \\
\hline 0.00 & & 3.5 & 1.55 & 101.3 & 99.75 \\
\hline 10.00 & 3.5 & & 1.51 & 101.3 & 99.79 \\
\hline 10.00 & & 3.5 & 1.48 & 101.3 & 99.82 \\
\hline 20.00 & 3.5 & & 1.58 & 101.3 & 99.72 \\
\hline 20.00 & & 3.5 & 1.48 & 101.3 & 99.82 \\
\hline 30.00 & 3.5 & & 1.58 & 101.3 & 99.72 \\
\hline 30.00 & & 3.5 & 1.5 & 101.3 & 99.8 \\
\hline 40.00 & 3.5 & & 1.6 & 101.3 & 99.7 \\
\hline 40.00 & & 3.5 & 1.55 & 101.3 & 99.75 \\
\hline 50.00 & 3.5 & & 1.52 & 101.3 & 99.78 \\
\hline 50.00 & & 3.5 & 1.49 & 101.3 & 99.81 \\
\hline 60.00 & 3.5 & & 1.62 & 101.3 & 99.68 \\
\hline 60.00 & & 3.5 & 1.31 & 101.3 & 99.99 \\
\hline 70.00 & 3.5 & & 1.55 & 101.3 & 99.75 \\
\hline 70.00 & & 3.5 & 1.52 & 101.3 & 99.78 \\
\hline 80.00 & 3.5 & & 1.49 & 101.3 & 99.81 \\
\hline 80.00 & & 3.5 & 1.56 & 101.3 & 99.74 \\
\hline 90.00 & 3.5 & & 1.6 & 101.3 & 99.7 \\
\hline 90.00 & & 3.5 & 1.7 & 101.3 & 99.6 \\
\hline 100.00 & 3.5 & & 1.58 & 101.3 & 99.72 \\
\hline & & & & & \\
\hline & & & & \\
\hline
\end{tabular}




\begin{tabular}{|c|c|c|c|c|c|}
\hline 100.00 & & 3.5 & 1.72 & 101.3 & 99.58 \\
\hline 110.00 & 3.5 & & 1.57 & 101.3 & 99.73 \\
\hline 110.00 & & 3.5 & 1.45 & 101.3 & 99.85 \\
\hline 120.00 & 3.5 & & 1.59 & 101.3 & 99.71 \\
\hline 120.00 & & 3.5 & 1.56 & 101.3 & 99.74 \\
\hline 130.00 & 3.5 & & 1.62 & 101.3 & 99.68 \\
\hline 130.00 & & 3.5 & 1.59 & 101.3 & 99.71 \\
\hline 140.00 & 3.5 & & 1.64 & 101.3 & 99.66 \\
\hline 140.00 & & 3.5 & 1.65 & 101.3 & 99.65 \\
\hline 150.00 & 3.5 & & 1.67 & 101.3 & 99.63 \\
\hline 150.00 & & 3.5 & 1.59 & 101.3 & 99.71 \\
\hline 160.00 & 3.5 & & 1.68 & 101.3 & 99.62 \\
\hline 160.00 & & 3.5 & 1.58 & 101.3 & 99.72 \\
\hline 170.00 & 3.5 & & 1.69 & 101.3 & 99.61 \\
\hline 170.00 & & 3.5 & 1.65 & 101.3 & 99.65 \\
\hline 180.00 & 3.5 & & 1.58 & 101.3 & 99.72 \\
\hline 180.00 & & 3.5 & 1.55 & 101.3 & 99.75 \\
\hline 190.00 & 3.5 & & 1.72 & 101.3 & 99.58 \\
\hline 190.00 & & 3.5 & 1.69 & 101.3 & 99.61 \\
\hline 200.00 & 3.5 & & 1.5 & 101.3 & 99.8 \\
\hline 200.00 & & 3.5 & 1.42 & 101.3 & 99.88 \\
\hline 210.00 & 3.5 & & 1.55 & 101.5 & 99.95 \\
\hline 210.00 & & 3.5 & 1.51 & 101.5 & 99.99 \\
\hline 220.00 & 3.5 & & 1.53 & 101.5 & 99.97 \\
\hline 220.00 & & 3.5 & 1.56 & 101.5 & 99.94 \\
\hline 230.00 & 3.5 & & 1.57 & 101.5 & 99.93 \\
\hline 230.00 & & 3.5 & 1.58 & 101.5 & 99.92 \\
\hline 240.00 & 3.5 & & 1.59 & 101.5 & 99.91 \\
\hline 240.00 & & 3.5 & 1.62 & 101.5 & 99.88 \\
\hline 250.00 & 3.5 & & 1.6 & 101.5 & 99.9 \\
\hline 250.00 & & 3.5 & 1.67 & 101.5 & 99.83 \\
\hline 260.00 & 3.5 & & 1.63 & 101.5 & 99.87 \\
\hline 260.00 & & 3.5 & 1.69 & 101.5 & 99.81 \\
\hline 270.00 & 3.5 & & 1.64 & 101.5 & 99.86 \\
\hline 270.00 & & 3.5 & 1.75 & 101.5 & 99.75 \\
\hline 280.00 & 3.5 & & 1.65 & 101.5 & 99.85 \\
\hline 280.00 & & 3.5 & 1.79 & 101.5 & 99.71 \\
\hline 290.00 & 3.5 & & 1.74 & 101.5 & 99.76 \\
\hline 290.00 & & 3.5 & 1.81 & 101.5 & 99.69 \\
\hline 300.00 & 3.5 & & 1.82 & 101.5 & 99.68 \\
\hline 300.00 & & 3.5 & 2 & 101.5 & 99.5 \\
\hline 310.00 & 3.5 & & 2.2 & 101.5 & 99.3 \\
\hline 310.00 & & 3.5 & 2.1 & 101.5 & 99.4 \\
\hline 320.00 & 3.5 & & 2.3 & 101.5 & 99.2 \\
\hline 320.00 & & 3.5 & 2.4 & 101.5 & 99.1 \\
\hline 330.00 & 3.5 & & 2.25 & 101.5 & 99.25 \\
\hline 330.00 & & 3.5 & 2.3 & 101.5 & 99.2 \\
\hline 340.00 & 3.5 & & 2.2 & 101.5 & 99.3 \\
\hline 340.00 & & 3.5 & 2.1 & 101.5 & 99.4 \\
\hline 350.00 & 3.5 & & 2.25 & 101.5 & 99.25 \\
\hline 350.00 & & 3.5 & 2.29 & 101.5 & 99.21 \\
\hline 360.00 & 3.5 & & 2.32 & 101.5 & 99.18 \\
\hline 360.00 & & 3.5 & 2.33 & 101.5 & 99.17 \\
\hline 370.00 & 3.5 & & 2.35 & 101.5 & 99.15 \\
\hline 370.00 & & 3.5 & 2.37 & 101.5 & 99.13 \\
\hline 380.00 & 3.5 & & 2.38 & 101.5 & 99.12 \\
\hline 380.00 & & 3.5 & 2.4 & 101.5 & 99.1 \\
\hline 390.00 & 3.5 & & 2.41 & 101.5 & 99.09 \\
\hline 390.00 & & 3.5 & 2.36 & 101.5 & 99.14 \\
\hline 400.00 & 3.5 & & 2.32 & 101.5 & 99.18 \\
\hline 400.00 & & 3.5 & 2.26 & 101.5 & 99.24 \\
\hline 410.00 & 3.5 & & 2.22 & 101.5 & 99.28 \\
\hline 410.00 & & 3.5 & 2.25 & 101.5 & 99.25 \\
\hline 420.00 & 3.5 & & 2.27 & 101.5 & 99.23 \\
\hline 420.00 & & 3.5 & 2.39 & 101.5 & 99.11 \\
\hline 430.00 & 3.5 & & 2.31 & 101.5 & 99.19 \\
\hline 430.00 & & 3.5 & 2.32 & 101.5 & 99.18 \\
\hline 440.00 & 3.5 & & 2.35 & 101.5 & 99.15 \\
\hline 440.00 & & 3.5 & 1.9 & 101.5 & 99.6 \\
\hline 450.00 & 3.5 & & 1.8 & 101.5 & 99.7 \\
\hline
\end{tabular}




\begin{tabular}{|c|c|c|c|c|c|}
\hline 450.00 & & 3.5 & 1.6 & 101.5 & 99.9 \\
\hline 460.00 & 3.5 & & 1.7 & 101.5 & 99.8 \\
\hline 460.00 & & 3.5 & 1.6 & 101.5 & 99.9 \\
\hline 470.00 & 3.5 & & 1.5 & 101.5 & 100 \\
\hline 470.00 & & 3.5 & 1.4 & 101.5 & 100.1 \\
\hline 480.00 & 3.5 & & 1.6 & 101.5 & 99.9 \\
\hline 480.00 & & 3.5 & 1.63 & 101.5 & 99.87 \\
\hline 490.00 & 3.5 & & 1.65 & 101.5 & 99.85 \\
\hline 490.00 & & 3.5 & 1.68 & 101.5 & 99.82 \\
\hline 500.00 & 3.5 & & 1.7 & 101.5 & 99.8 \\
\hline 500.00 & & 3.5 & 1.62 & 101.5 & 99.88 \\
\hline
\end{tabular}

\section{Analysis and Discussion}

The data collected from the field has been processed in different directions by considering the objectives of present study, they are

\section{i. Fixation of formation level along the alignment}

The R.L's of existing ground in longitudinal sectioning has been computed by "Height of Instrument" method and is present in chapter 3 tables.

\section{ii. Compute the gradient and R.L's of the formation levels}

The computation of gradient and R.L's of the formation level from $0 \mathrm{~m}$ to $(250 \mathrm{~m}+250 \mathrm{~m}+250 \mathrm{~m}) 750 \mathrm{~m}$ chainage is as follows.

Effective gradient $=($ R.L of highest point-R.L of lowest point $) /$ length of stretch.

\section{Gradient and Formation Levels Of Road:}

Table 3- Formation level of road

\begin{tabular}{|c|c|c|c|c|}
\hline $\begin{array}{c}\text { CHAIN } \\
\text { AGE }\end{array}$ & $\begin{array}{c}\text { EXISTING } \\
\text { GROUND } \\
\text { LEVEL }\end{array}$ & $\begin{array}{c}\text { FL= } \\
\text { R.L-Z }\end{array}$ & $\begin{array}{c}\text { NATURE } \\
\text { (FALL/RISE) }\end{array}$ & $\begin{array}{l}\text { EFFECTIVE } \\
\text { GRADIENT }\end{array}$ \\
\hline & 100 & 99.949 & Rise & 0.051 \\
\hline 0.00 & 99.49 & 99.451 & Rise & 0.039 \\
\hline 10.00 & 99.1 & 99.081 & Rise & 0.019 \\
\hline 20.00 & 98.91 & 98.94 & Fall & -0.03 \\
\hline 30.00 & 99.21 & 99.211 & Fall & -0.001 \\
\hline 40.00 & 99.22 & 99.227 & Fall & -0.007 \\
\hline 50.00 & 99.29 & 99.272 & Rise & 0.018 \\
\hline 60.00 & 99.11 & 99.109 & Rise & 0.001 \\
\hline 70.00 & 99.1 & 99.178 & Fall & -0.078 \\
\hline 80.00 & 99.88 & 99.872 & Rise & 0.008 \\
\hline 90.00 & 99.8 & 99.801 & Fall & -0.001 \\
\hline 100.00 & 99.81 & 99.802 & Rise & 0.008 \\
\hline 110.00 & 99.73 & 99.728 & Rise & 0.002 \\
\hline 120.00 & 99.71 & 99.707 & Rise & 0.003 \\
\hline 130.00 & 99.68 & 99.678 & Rise & 0.002 \\
\hline 140.00 & 99.66 & 99.659 & Rise & 0.001 \\
\hline 150.00 & 99.65 & 99.649 & Rise & 0.001 \\
\hline 160.00 & 99.64 & 99.638 & Rise & 0.002 \\
\hline 170.00 & 99.62 & 99.632 & Fall & -0.012 \\
\hline 180.00 & 99.74 & 99.744 & Fall & -0.004 \\
\hline 190.00 & 99.78 & 99.782 & Fall & -0.002 \\
\hline 200.00 & 99.8 & 99.82 & Fall & -0.02 \\
\hline 210.00 & 100 & 99.996 & Rise & 0.004 \\
\hline 220.00 & 99.96 & 99.959 & Rise & 0.001 \\
\hline 230.00 & 99.95 & 99.948 & Rise & 0.002 \\
\hline 240.00 & 99.93 & 99.928 & Rise & 0.002 \\
\hline 250.00 & 99.91 & 99.909 & Rise & 0.001 \\
\hline 260.00 & 99.9 & 99.897 & Rise & 0.003 \\
\hline 270.00 & 99.87 & 99.869 & Rise & 0.001 \\
\hline 280.00 & 99.86 & 99.859 & Rise & 0.001 \\
\hline 290.00 & 99.85 & 99.841 & Rise & 0.009 \\
\hline 300.00 & 99.76 & 99.752 & Rise & 0.008 \\
\hline 310.00 & 99.68 & 99.641 & Rise & 0.039 \\
\hline 320.00 & 99.29 & 99.279 & Rise & 0.011 \\
\hline 330.00 & 99.18 & 99.179 & Rise & 0.001 \\
\hline 340.00 & 99.17 & 99.183 & Fall & -0.013 \\
\hline 350.00 & 99.3 & 99.295 & Rise & 0.005 \\
\hline 360.00 & 99.25 & 99.243 & Rise & 0.007 \\
\hline 370.00 & 99.18 & 99.177 & Rise & 0.003 \\
\hline
\end{tabular}




\begin{tabular}{|c|c|c|c|c|}
\hline 380.00 & 99.15 & 99.149 & Rise & 0.001 \\
\hline 390.00 & 99.14 & 99.138 & Rise & 0.002 \\
\hline 400.00 & 99.12 & 99.117 & Rise & 0.003 \\
\hline 410.00 & 99.09 & 99.099 & Fall & -0.009 \\
\hline 420.00 & 99.18 & 99.19 & Fall & -0.01 \\
\hline 430.00 & 99.28 & 99.275 & Rise & 0.005 \\
\hline 440.00 & 99.23 & 99.226 & Rise & 0.004 \\
\hline 450.00 & 99.19 & 99.186 & Rise & 0.004 \\
\hline 460.00 & 99.15 & 99.205 & Fall & -0.055 \\
\hline 470.00 & 99.7 & 99.71 & Fall & -0.01 \\
\hline 480.00 & 99.8 & 99.82 & Fall & -0.02 \\
\hline 490.00 & 100 & 100 & Rise & 0 \\
\hline 500.00 & 100 & 99.99 & Rise & 0.01 \\
\hline
\end{tabular}

\section{Depth of cut and fill for road:}

\begin{tabular}{|c|c|c|c|c|c|c|}
\hline $\begin{array}{c}\text { CHAI } \\
\text { NAGE }\end{array}$ & H.I & $\begin{array}{c}\text { R.L of } \\
\text { Formation }\end{array}$ & CUT & FILL & $\begin{array}{l}\text { Observed } \\
\text { Staff } \\
\text { Reading }\end{array}$ & $\begin{array}{c}\text { Staff } \\
\text { Reading To Be } \\
\text { Set }\end{array}$ \\
\hline & & & & & 1.3 & \\
\hline 0.00 & 101.30 & 99.949 & 0.46 & & 1.81 & 1.35 \\
\hline 10.00 & 101.30 & 99.451 & 0.35 & & 2.20 & 1.85 \\
\hline 20.00 & 101.30 & 99.081 & 0.17 & & 2.39 & 2.22 \\
\hline 30.00 & 101.30 & 98.94 & & -0.05 & 2.31 & 2.36 \\
\hline 40.00 & 101.30 & 99.211 & 0.00 & & 2.09 & 2.09 \\
\hline 50.00 & 101.30 & 99.227 & 0.01 & & 2.08 & 2.07 \\
\hline 60.00 & 101.30 & 99.272 & & -0.02 & 2.01 & 2.03 \\
\hline 70.00 & 101.30 & 99.109 & -0.18 & & 2.01 & 2.19 \\
\hline 80.00 & 101.30 & 99.178 & 0.08 & & 2.20 & 2.12 \\
\hline 90.00 & 101.30 & 99.872 & & -0.01 & 1.42 & 1.43 \\
\hline 100 & 101.30 & 99.801 & 0.00 & & 1.50 & 1.50 \\
\hline 110 & 101.30 & 99.802 & 0.07 & & 1.57 & 1.50 \\
\hline 120 & 101.30 & 99.728 & 0.02 & & 1.59 & 1.57 \\
\hline 130 & 101.30 & 99.707 & 0.03 & & 1.62 & 1.59 \\
\hline 140 & 101.30 & 99.678 & 0.02 & & 1.64 & 1.62 \\
\hline 150 & 101.30 & 99.659 & 0.01 & & 1.65 & 1.64 \\
\hline 160 & 101.30 & 99.649 & 0.01 & & 1.66 & 1.65 \\
\hline 170 & 101.30 & 99.638 & 0.02 & & 1.68 & 1.66 \\
\hline 180 & 101.30 & 99.632 & & -0.11 & 1.56 & 1.67 \\
\hline 190 & 101.30 & 99.744 & & -0.04 & 1.52 & 1.56 \\
\hline 200 & 101.30 & 99.782 & & -0.02 & 1.50 & 1.52 \\
\hline 210 & 101.30 & 99.82 & 0.02 & & 1.50 & 1.48 \\
\hline 220.00 & 101.50 & 99.996 & 0.04 & & 1.54 & 1.50 \\
\hline 230.00 & 101.50 & 99.959 & 0.01 & & 1.55 & 1.54 \\
\hline 240.00 & 101.50 & 99.948 & 0.02 & & 1.57 & 1.55 \\
\hline 250.00 & 101.50 & 99.928 & 0.02 & & 1.59 & 1.57 \\
\hline 260.00 & 101.50 & 99.909 & 0.01 & & 1.60 & 1.59 \\
\hline 270.00 & 101.50 & 99.897 & 0.03 & & 1.63 & 1.60 \\
\hline 280.00 & 101.50 & 99.869 & 0.01 & & 1.64 & 1.63 \\
\hline 290.00 & 101.50 & 99.859 & 0.01 & & 1.65 & 1.64 \\
\hline 300.00 & 101.50 & 99.841 & 0.08 & & 1.74 & 1.66 \\
\hline 310.00 & 101.50 & 99.752 & 0.07 & & 1.82 & 1.75 \\
\hline 320.00 & 101.50 & 99.641 & 0.35 & & 2.21 & 1.86 \\
\hline 330.00 & 101.50 & 99.279 & 0.10 & & 2.32 & 2.22 \\
\hline 340.00 & 101.50 & 99.179 & 0.01 & & 2.33 & 2.32 \\
\hline 350.00 & 101.50 & 99.183 & & -0.12 & 2.20 & 2.32 \\
\hline 360.00 & 101.50 & 99.295 & 0.05 & & 2.25 & 2.21 \\
\hline 370.00 & 101.50 & 99.243 & 0.06 & & 2.32 & 2.26 \\
\hline 380.00 & 101.50 & 99.177 & 0.03 & & 2.35 & 2.32 \\
\hline 390.00 & 101.50 & 99.149 & 0.01 & & 2.36 & 2.35 \\
\hline 400.00 & 101.50 & 99.138 & 0.02 & & 2.38 & 2.36 \\
\hline 410.00 & 101.50 & 99.117 & 0.03 & & 2.41 & 2.38 \\
\hline 420.00 & 101.50 & 99.099 & & -0.08 & 2.32 & 2.40 \\
\hline 430.00 & 101.50 & 99.19 & & -0.09 & 2.22 & 2.31 \\
\hline 440.00 & 101.50 & 99.275 & 0.05 & & 2.27 & 2.22 \\
\hline 450.00 & 101.50 & 99.226 & 0.04 & & 2.31 & 2.27 \\
\hline 460.00 & 101.50 & 99.186 & 0.04 & & 2.35 & 2.31 \\
\hline 470.00 & 101.50 & 99.205 & & -0.5 & 1.80 & 2.30 \\
\hline 480.00 & 101.50 & 99.71 & & -0.09 & 1.70 & 1.79 \\
\hline
\end{tabular}




\begin{tabular}{|l|c|c|c|c|c|c|}
\hline 490.00 & 101.50 & 99.82 & & -0.18 & 1.50 & 1.68 \\
\hline 500.00 & 101.50 & 100 & 0.00 & & 1.50 & 1.50 \\
\hline
\end{tabular}

Earthwork Calculations:

Table 5- Earthwork Calculations for Road:

\begin{tabular}{|c|c|c|c|c|c|c|}
\hline HEIGHT & $\begin{array}{c}\text { MEAN } \\
\text { HT. }\end{array}$ & $\begin{array}{c}\text { CENTRAL } \\
\text { AREA } \\
\text { BD }\end{array}$ & $\begin{array}{l}\text { AREA } \\
\text { OF } \\
\text { SIDES } \\
\text { SD }^{2}\end{array}$ & $\begin{array}{c}\text { TOTAL } \\
\text { SECTIONAL } \\
\text { AREA }\left(\text { BD+SD }^{2}\right)\end{array}$ & $\begin{array}{c}\text { DISTANCE } \\
\text { IN } \\
\text { BETWEEN } \\
\text { STATION } \\
\text { L }\end{array}$ & $\begin{array}{l}\text { QUANTITY } \\
\text { (BD+SD'L) }\end{array}$ \\
\hline-0.46 & -0.405 & -2.835 & 0.246038 & -2.5889625 & 10 & -0.374625 \\
\hline-0.35 & -0.26 & -1.82 & 0.1014 & -1.7186 & 10 & -0.806 \\
\hline-0.17 & -0.06 & -0.42 & 0.0054 & -0.4146 & 10 & -0.366 \\
\hline 0.05 & 0.025 & 0.175 & 0.000938 & 0.1759375 & 10 & 0.184375 \\
\hline 0 & -0.005 & -0.035 & $3.75 \mathrm{E}-05$ & -0.0349625 & 10 & -0.034625 \\
\hline-0.01 & 0.005 & 0.035 & $3.75 \mathrm{E}-05$ & 0.0350375 & 10 & 0.035375 \\
\hline 0.02 & 0.01 & 0.07 & 0.00015 & 0.07015 & 10 & 0.0715 \\
\hline 0 & -0.04 & -0.28 & 0.0024 & -0.2776 & 10 & -0.256 \\
\hline-0.08 & -0.035 & -0.245 & 0.001838 & -0.2431625 & 10 & -0.226625 \\
\hline 0.01 & 0.005 & 0.035 & $3.75 \mathrm{E}-05$ & 0.0350375 & 10 & 0.035375 \\
\hline 0 & -0.035 & -0.245 & 0.001838 & -0.2431625 & 10 & -0.226625 \\
\hline-0.07 & -0.045 & -0.315 & 0.003038 & -0.3119625 & 10 & -0.284625 \\
\hline-0.02 & -0.025 & -0.175 & 0.000938 & -0.1740625 & 10 & -0.165625 \\
\hline-0.03 & -0.025 & -0.175 & 0.000938 & -0.1740625 & 10 & -0.165625 \\
\hline-0.02 & -0.015 & -0.105 & 0.000338 & -0.1046625 & 10 & -0.101625 \\
\hline-0.01 & -0.01 & -0.07 & 0.00015 & -0.06985 & 10 & -0.0685 \\
\hline-0.01 & -0.015 & -0.105 & 0.000338 & -0.1046625 & 10 & -0.101625 \\
\hline-0.02 & 0.045 & 0.315 & 0.003038 & 0.3180375 & 10 & 0.345375 \\
\hline 0.11 & 0.075 & 0.525 & 0.008438 & 0.5334375 & 10 & 0.609375 \\
\hline 0.04 & 0.03 & 0.21 & 0.00135 & 0.21135 & 10 & 0.2235 \\
\hline 0.02 & 0 & 0 & 0 & 0 & 10 & 0 \\
\hline-0.02 & -0.03 & -0.21 & 0.00135 & -0.20865 & 10 & -0.1965 \\
\hline-0.04 & -0.025 & -0.175 & 0.000938 & -0.1740625 & 10 & -0.165625 \\
\hline-0.01 & -0.015 & -0.105 & 0.000338 & -0.1046625 & 10 & -0.101625 \\
\hline-0.02 & -0.02 & -0.14 & 0.0006 & -0.1394 & 10 & -0.134 \\
\hline-0.02 & -0.015 & -0.105 & 0.000338 & -0.1046625 & 10 & -0.101625 \\
\hline-0.01 & -0.02 & -0.14 & 0.0006 & -0.1394 & 10 & -0.134 \\
\hline-0.03 & -0.02 & -0.14 & 0.0006 & -0.1394 & 10 & -0.134 \\
\hline-0.01 & -0.01 & -0.07 & 0.00015 & -0.06985 & 10 & -0.0685 \\
\hline-0.01 & -0.045 & -0.315 & 0.003038 & -0.3119625 & 10 & -0.284625 \\
\hline-0.08 & -0.075 & -0.525 & 0.008438 & -0.5165625 & 10 & -0.440625 \\
\hline-0.07 & -0.21 & -1.47 & 0.06615 & -1.40385 & 10 & -0.8085 \\
\hline-0.35 & -0.225 & -1.575 & 0.075938 & -1.4990625 & 10 & -0.815625 \\
\hline-0.10 & -0.055 & -0.385 & 0.004538 & -0.3804625 & 10 & -0.339625 \\
\hline-0.01 & 0.055 & 0.385 & 0.004538 & 0.3895375 & 10 & 0.430375 \\
\hline 0.12 & 0.035 & 0.245 & 0.001838 & 0.2468375 & 10 & 0.263375 \\
\hline-0.05 & -0.055 & -0.385 & 0.004538 & -0.3804625 & 10 & -0.339625 \\
\hline-0.06 & -0.045 & -0.315 & 0.003038 & -0.3119625 & 10 & -0.284625 \\
\hline-0.03 & -0.02 & -0.14 & 0.0006 & -0.1394 & 10 & -0.134 \\
\hline-0.01 & -0.015 & -0.105 & 0.000338 & -0.1046625 & 10 & -0.101625 \\
\hline-0.02 & -0.025 & -0.175 & 0.000938 & -0.1740625 & 10 & -0.165625 \\
\hline-0.03 & 0.025 & 0.175 & 0.000938 & 0.1759375 & 10 & 0.184375 \\
\hline 0.08 & 0.085 & 0.595 & 0.010838 & 0.6058375 & 10 & 0.703375 \\
\hline 0.09 & 0.02 & 0.14 & 0.0006 & 0.1406 & 10 & 0.146 \\
\hline-0.05 & -0.045 & -0.315 & 0.003038 & -0.3119625 & 10 & -0.284625 \\
\hline-0.04 & -0.04 & -0.28 & 0.0024 & -0.2776 & 10 & -0.256 \\
\hline-0.04 & 0.23 & 1.61 & 0.07935 & 1.68935 & 10 & 2.4035 \\
\hline 0.50 & 0.295 & 2.065 & 0.130538 & 2.1955375 & 10 & 3.370375 \\
\hline 0.09 & 0.135 & 0.945 & 0.027338 & 0.9723375 & 10 & 1.218375 \\
\hline 0.18 & 0.09 & 0.63 & 0.01215 & 0.64215 & 10 & 0.7515 \\
\hline
\end{tabular}

\section{Conclusion and Scope of Further Study}

\section{Conclusion:}

In the present study an attempt has been made to establish the alignment of a highway of length $1 \mathrm{~km}$ from "Huzurabad to Parkal" for extension of highway from two way lane to four way lane and evaluation of soil strength on the field in the form of an open transverse. The road is categorized as a village road. Detailed profile levelling has been aimed out along the alignment; the formation level of the road has been fixed according to the prevailing topography. 


\section{Scope of Further Study:}

In the present study as the computers are taking centre stage in almost all the civil engineering projects. It can be used for the most optimum highway design consisting of

1. The horizontal alignment

2. Vertical alignment

3. Road cross-section

4. Super elevation

5. Computation of earthworks.

The engineer can now consider various alternatives and evaluate them so as to produce the most economical option. Great savings in time is possible and the cost of engineering can thus be reduced very much. Instead of the conventional circular curves with two transitional ends it is now possible to design. The digital terrain mode (DTM) enables evaluation of a number of alternative vertical and horizontal alignments and selects the best and gives the quantities of earthwork. Computers can develop perspective views of the alignment so that the one, which is the most aesthetically pleasing and gives comfort and safety can be selected. The computer can work out the ground cross section and road cross section and give them as printouts.

\section{Bibliography}

[1] Khanna S.K \& Justo C.E.G, "Highway Engineering". New Chand \& Bros., Roorkee, U.K India, 9th Edition:2011

[2] S.K Duggal, " Surveying" Tata McGraw Hill Education Pvt.Ltd, 3rd Edition:2009

[3] Yoder \&Witczak, "Pavement Design"

[4] Mannering \& Kilareski, "Traffic Engineering \& Pavement Design"

[5] B.C.Punmia "Surveying" Vol. 1 \& 2 